\title{
Probleme der Wasserversorgung von La Paz (Bolivien)
}

\section{Geographischer Rahmen}

Besucher von La Paz wundern sich oft, daß diese Stadt ausgerechnet in einem zerfurchten Tal zwischen Altiplano und Kordillere gebaut worden ist. Einen Anlaß zur Gründung gaben den Indios die Goldseifen des Río Choqueyapu (Gold heißt in der Aymarasprache «chocque»). Ebenso wichtig war das Angebot an Trinkwasser, denn dieser Fluß führt ständig Wasser, im Gegensatz zu vielen Bächen auf dem Altiplano. Ein Beispiel dafür ist der Río Seco (der "trockene Fluß») am heutigen Westrand der Stadt. Auch temperaturmäßig ist das Valle de $\mathrm{La} \mathrm{Paz}$ gegenüber dem Altiplano begünstigt (siehe Fig. 1). Hier sinkt die Temperatur in der winterlichen Trokkenzeit häufig unter den Gefrierpunkt, während dies im Stadtzentrum nur in besonders kalten Jahren vorkommt. Dies erkannten auch die spanischen Kolonisatoren, denn sie gründeten 1548 ihre Siedlung «Ciudad de nuestra virgen de la Paz» im Talkessel und nicht auf dem Altiplano, obschon hier die wichtige Erzhandelsstraße Lima-Potosí durchzog. Abgesehen davon genießt $\mathrm{La} \mathrm{Paz}$ etliche Lagevorteile: Hier vereinen sich die Routen aus den Früchteanbaugebieten im NE und SE, und die Stadt liegt an einem strategisch wichtigen Durchgang vom Altiplano zum Tiefland. Die Agglomeration zählt ca. 650000 Einwohner (1976, nach Angaben des Instituto Nacional de Estadística, zit. in SCHOop 1980, S. 219).

Die heutige Wasserversorgung von La Paz muß den geographischen Gegebenheiten Rechnung tragen. Dank der nahen Gletscher der Cordillera Real kann man viel Schmelzwasser nutzen'. Zudem erübrigt das natürliche Gefälle den Bau kostspieliger Pumpwerke. Gleichzeitig stellen sich dabei aber große Probleme. Die Stadt erstreckt sich nämlich über ein Höhenintervall von annähernd $1000 \mathrm{~m}$; heute klettern die obersten Häuser auf dem Altiplano bis $4180 \mathrm{~m}$, die tiefstgelegenen sind auf $3230 \mathrm{~m}$. Somit muß der Wasserdruck je nach Höhenlage der Quartiere reduziert werden. Zudem wirken Erosion und Denudation so intensiv, daß es oft zu Brüchen oder Verstopfungen von Wasser- und Abwasserleitungen kommt. Auch sind in Lagen oberhalb $4000 \mathrm{~m}$ die Leitungen an Wintermorgen häufig zugefroren. Die lagemäßige und klimatische Benachteiligung der Quartiere auf dem Altiplano bringt es mit sich, daß hier finanziell schlecht gestellte Leute wohnen. Dagegen liegen die
Wohngebiete reicher Familien im allgemeinen weit unten. Dieser soziale Gegensatz äußert sich auch in sehr unterschiedlichen Verhältnissen des Wasserverbrauchs. Aus all diesen Gründen gestaltet sich die Wasserversorgung der Stadt schwierig ${ }^{2}$. Sie ist uneinheitlich und komplex, da sogar innerhalb der Stadtteile verschiedene Regelungen getroffen worden sind. Ziel dieser Studie war es, den Entwicklungsstand der Infrastruktur am Beispiel der Wasserversorgung abzutesten und Möglichkeiten der Verbesserung aufzuzeigen $^{3}$.

\section{Systeme der Wasserversorgung in La Paz}

\section{1 Überblick}

Das Gesamtbild der Wasserversorgung wird durch ein 1966 für La Paz entworfenes Grundkonzept geprägt (косKs 1967). Es entstand damals als Leitbild bei der Gründung des städtischen Amtes für Trinkwasser und Kanalisation, SAMAPA ( Servicio Autónomo Municipal de Agua Potable y Alcantorillado»). Zwei weitere Amtsstellen beteiligen sich an der Wasserversorgung, nämlich die staatliche Wohnungsbaugenossenschaft CONAVI («Corporación Nacional de Vivienda») und die Stadtverwaltung («Honorable Alcaldía Municipal»). Daneben sind zahlreiche private Genossenschaften tätig, und in anderen Fällen versorgen sich Haushalte selbst mit Wasser, sei dies aus Quellen, Brunnen oder Bächen.

Die Qualität des Wassers hängt natürlich sehr von den Versorgungssystemen ab. SAMAPA liefert gefiltertes und z.T. mit Chlor aufbereitetes Wasser. Die Benützung anderen Wassers ist an einige Voraussetzungen geknüpft. Einheimische besitzen gegen mehrere Krankheiten (Gelbsucht, Darminfektion) eigene Abwehrstoffe. Sie haben sich mit der Verwendung verschmutzten Wassers abgehärtet, wobei die Kindersterblichkeit groß ist $(17 \% / \mathrm{Jahr}$ nach WELTBANK 1979 , zit. in sсноор 1980, S. 8). Im weiteren pflegen die Frauen z. B. einen Suppen-Eintopf stundenlang zu

Dr. Kurt Graf, Geographisches Institut der Universität Zürich, Postfach, 8033 Zürich 


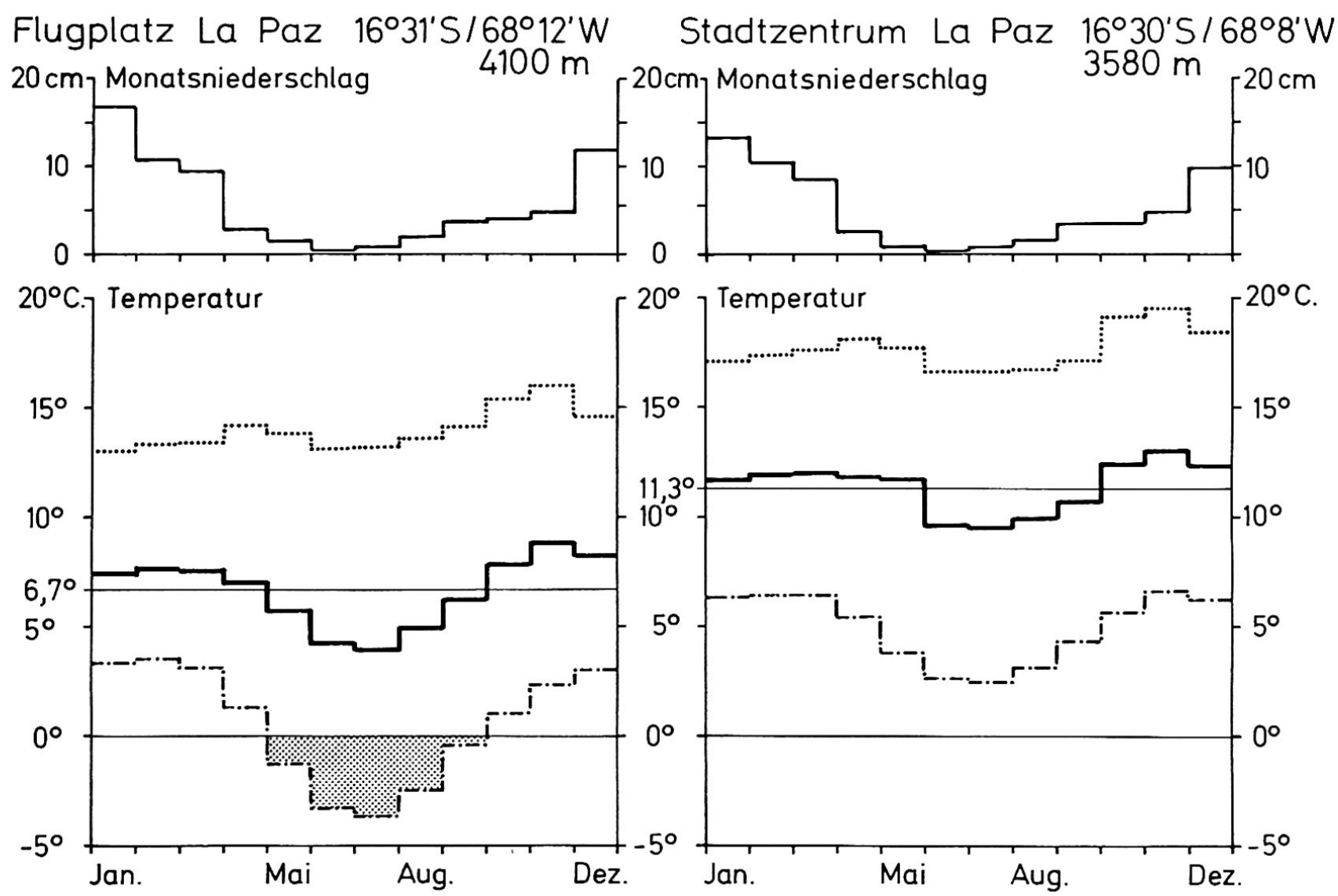

Fig. 1 Klimadiagramme zweier Stationen in La Paz (1972-1979, nach Daten der ANUARIOS METEOROLOGICOS, Servicio Nacional de Meteorologia e Hidrología, La Paz). Die Monatsmitteltemperaturen sind dreifach aufgetragen, nämlich der Durchschnitt (dick ausgezogen), das mittlere Maximum (punktiert) und das mittlere Minimum (strichpunktiert). Der Punktraster bezeichnet die Zeit häufiger Nachtfröste.

kochen, was die Gefahr bakterieller Ansteckung mindert. Die schlechte Wasserqualität kann also wegen einiger Gewohnheiten nicht mit europäischen Maßstäben gemessen werden.

Unsere Feldbegehungen und Befragungen erstreckten sich auf August und September 1981. An einigen Tagen stand ein Jeep des «Servicio Geológico de Bolivia» (GEOBOL) zur Verfügung, meistens aber war zur genaueren Kartierung das Abwandern der wichtigsten Achsen und Grenzsäume unumgänglich. Es zeigte sich nämlich bald, daß etliche Systeme der Wasserversorgung eng nebeneinander vorkommen oder sich sogar räumlich überlagern. Diese überraschend vielfältigen Arten werden im folgenden geschildert.

\subsection{SAMAPA}

Dieses Wasseramt wurde mit deutscher Hilfe geschaffen, der Kreditanstalt für Wiederaufbau, Frankfurt ${ }^{4}$. In zwei Etappen wurden ab 1971 und 1979 große Teile der Stadt mit Wasser aus nahegelegenen Stauseen versorgt (s. Fig. 2). Die Nutzung umfaßt zu rund $95 \%$ solches Gletscherschmelzwasser und Regenwasser und nur zu 5\% Wasser aus dem Río Choqueyapu, das v. a. im September bis November beigezogen wird ${ }^{5}$. Als Zuleitungen dienen schmale Kanäle, Aquädukte, Tunnels und Rohrleitungen ${ }^{6}$. In den drei Aufbereitungsanlagen El Alto, Achachicala und Pampahasi wird das Wasser gesäubert. Von dort aus führen Hauptleitungen in die drei Versorgungszonen der Stadt: El Alto (A in Fig. 2), das Stadtzentrum i.w. S. (B) und die von Pampahasi belieferten Stadtteile (C). Die Grenze von A und B folgt ungefähr der $3750 \mathrm{~m}$ Isohypse.

SAMAPA hat vorwiegend in den Zonen B und C Hausanschlüsse angebracht und garantiert einen durchschnittlichen Wasserdruck von 2 atü. Je nach Quartier werden Wasserzähler installiert oder Pauschalen erhoben. In Hochhäusern der City muß der Besitzer einen Tank im Keller, einen Tank auf dem Dach und dazwischen eine Pumpe installieren, damit Wasser in alle Stockwerke geführt werden kann. Anstelle von Hausanschlüssen baute SAMAPA v.a. in der Zone A öffentliche Brunnen (Abb. 1). Wer die Pauschale bezahlt, darf dort Wasser holen? ${ }^{7}$. Brunnen mit ständig fließendem Wasser und Gratisbezug von Wasser gibt es im ganzen Stadtgebiet nicht. Wie 


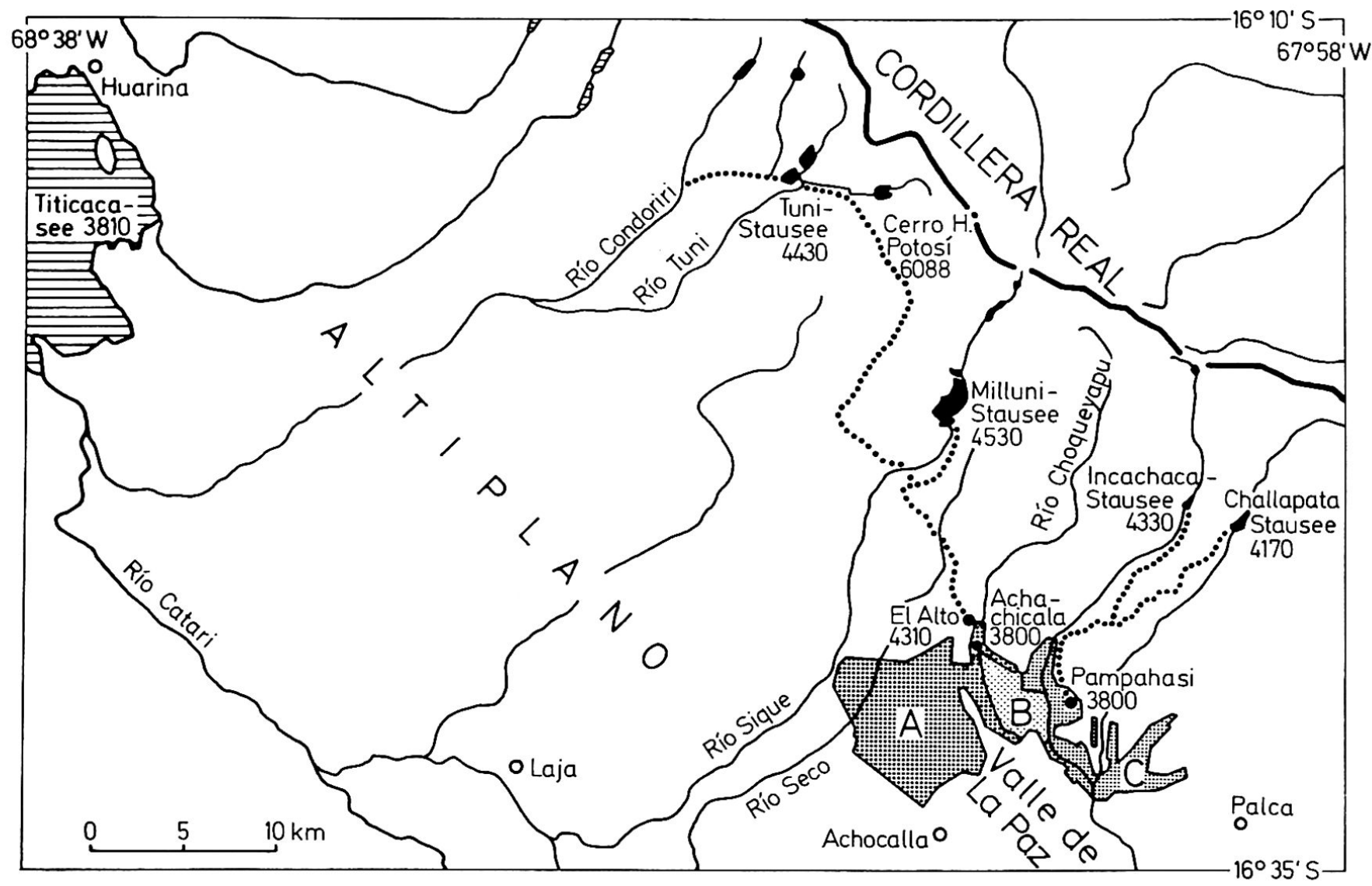

Fig. 2 Überblick zur Wasserversorgung von La Paz. Zahlreiche Berge wie der Cerro Huayna Potosi in der Cordillera Real sind vergletschert. Schmelz- und Regenwasser, das sich in den schwarz dargestellten Seen sammelt, wird in schmalen Kanälen (punktiert) nach La Paz geleitet. Die übrigen Seen sind schraffiert wiedergegeben, Höhenangaben sind in $m$ ü. M. Am nördlichen Rand von La Paz finden sich die Aufbereitungsanlagen El Alto, Achachicala und Pampahasi (schwarze Kreise). Sie beliefern die drei Versorgungszonen A, B und C mit Wasser. Grundlagen zu dieser Karte: SAMAPA-Plan 1:10 000 von NAVARRO (1980), Landnutzungsplan 1:10 000 von GALOPPO (1979) und eigene Erhebungen.

unterschiedlich das Angebot an Wasser in den einzelnen Stadtteilen ausfällt, zeigt Fig. 3. Ob diesem Angebot auch eine unterschiedliche Nachfrage zugrunde liegt, wird ein Diskussionspunkt in Kap. 3 sein.

\subsection{CONAVI}

Das nationale Wohnungsbauamt CONAVI errichtet Einfamilienhaussiedlungen und verkauft sie gegen Abzahlung. So entstand das «Barrio Autopista» (Koordinaten $80.5 / 91.3)^{8}$ für Hausbesitzer, die im Zuge des Autobahnbaus (1977-1980) enteignet wurden. Die Belieferung mit Wasser ist meistens ungenügend. Im Fall der Urbanisation "CONAVI Río Seco» (76.5/84.5) versieht ein Wasserturm (Abb.2) die Häuser halbtags mit Wasser. Die Leute sehen sich gezwungen, zusätzlich Wasser faßweise zu kaufen (s. Kap.4.2.). In den Häusern des Barrio Autopista fließt das Wasser nur in knapp bemessenen Intervallen aus einem Tank. In festem Rhythmus kann z.B. 15 Minuten Wasser geliefert werden und jeweils 30 Minuten abgestellt bleiben.

\subsection{Zisternenwagen der Stadtverwaltung}

In einzelnen Stadtteilen verkehren Tanklastwagen der Stadtverwaltung und füllen bereitstehende Blechfässer. Sie bringen aufbereitetes Wasser des SAMAPA und verkaufen es für ca. USS 1.- pro Faß (à $\left.1 \mathrm{~m}^{3}\right)$. Ihre Fahrpläne sind unterschiedlich, können die Zulieferungen doch von zweimal täglich (in CONAVI Río Seco) bis zweimal wöchentlich (im oberen Teil von Villa Fátima, 79.5/94.5) schwanken. Notfallmäßig können sie z.B. bei Hauptleitungsbrüchen in jedem Quartier eingesetzt werden. Im allgemeinen stellen aber die Zisternenwagen doch eine - auf mehrere Jahre - behelfsmäßige Wasserversorgung von Armenvierteln dar. Gegen Ende der Trockenzeit kann es hier im Kampf um die Wasserzuteilung gelegentlich zu Schlägereien kommen.

\subsection{Genossenschaften auf privater Basis}

Eine Form gemeinsamer Wasserversorgung stellen Kooperativen dar $^{9}$. 20 bis 50 Haushalte schlossen sich zusammen und bestimmten ein Führungsgremium 


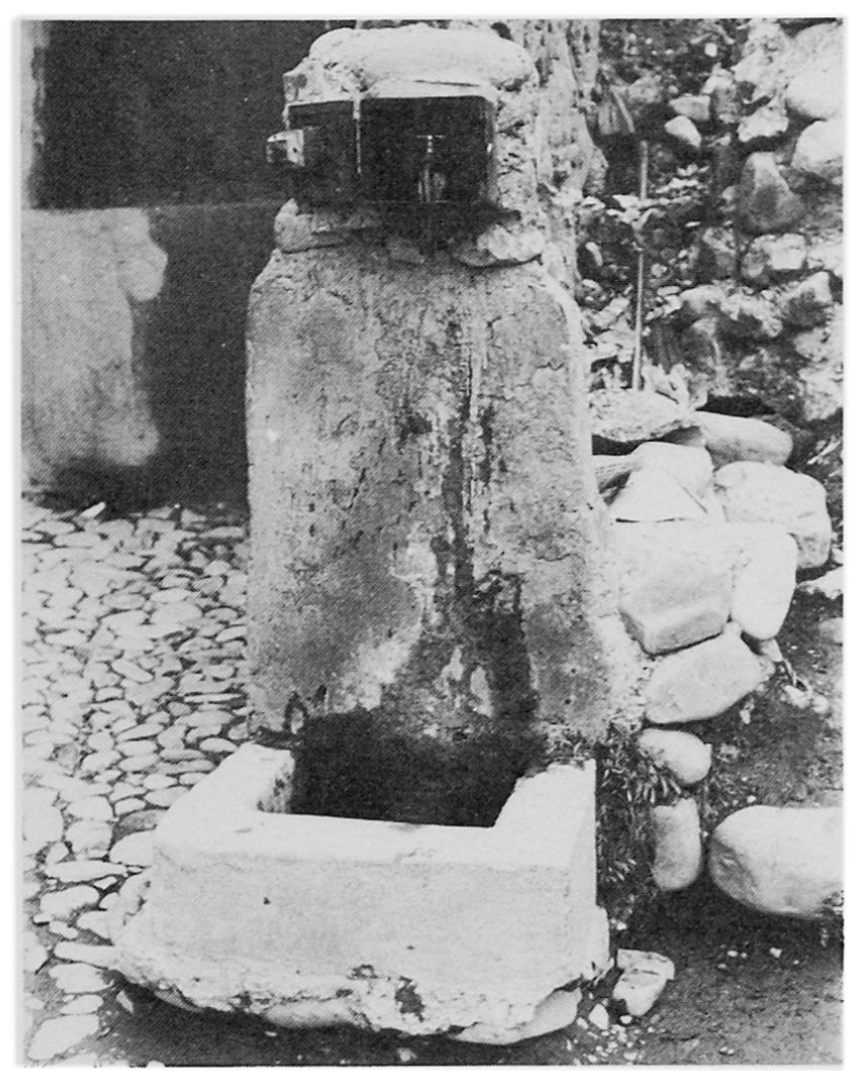

Abb.1 Öffentlicher Brunnen mit Schließkasten, 1,5 km nördlich der Plaza San Francisco (77.5/92.5).

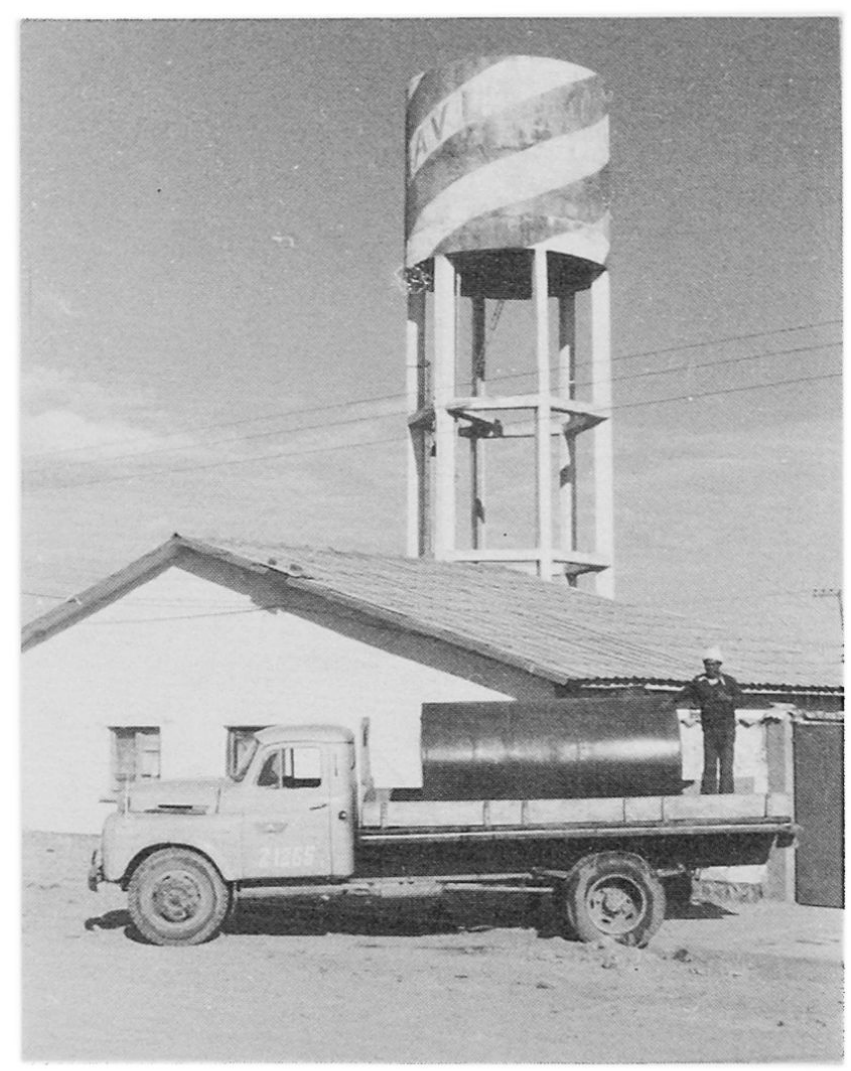

Abb. 2 Zisternenwagen und Wasserturm, die den Stadtteil CONAVI Rio Seco (76.5/84.5) mit Trinkwasser versorgen. mit einem Präsidenten. Sie faßten gemeinsam Quellen an den oberen Talflanken und installierten Tanks aus Blech oder Stein (Abb. 3) und ein Verteilnetz. Reparaturen und Erweiterungen werden in Fronarbeit vorgenommen. Diese Selbsthilfe in der Wasserversorgung funktioniert im ganzen Stadtteil «Las Lomas» (73/92.5). In Villa Fátima (79.5/95) arbeiten z. B. zwei Genossenschaften zusammen, und jede erhält während zwei Stunden alternierend Wasser. In Cota Cota (71/101) bestanden drei große Kooperativen, und als 1969 SAMAPA dorthin eine Hauptleitung fertiggestellt hatte, begannen Verhandlungen über die allmähliche Einstellung der privaten Organisationen. Auch das «Barrio ferroviario» (80.5/91) kannte früher Genossenschaftstanks, ebenso das Gebiet zwischen Miraflores und Pampahasi (76/95). Es sind Fälle bekannt, wo sich innerhalb einer Kooperative einzelne Gruppen rivalisieren und sich die einen den Tank aneignen. Dann kommen Wasserentzug und Erpressung vor. Diese Entwicklung wird geschürt durch die Bevölkerungszunahme und die meistens zu kleine Konzeption der Tanks. In einer ersten Sanierungsphase übernimmt SAMAPA das bestehende Verteilnetz weitgehend, ersetzt aber den Tank durch eine Hauptleitung.

\subsection{Private Brunnen und Quellen}

Oft besitzen Haushalte und Industriebetriebe eine eigene Wasserversorgung, die an besondere Voraussetzungen gebunden ist. So tritt in einem Teil von Villa Fátima (79.4/94.7) Quellwasser zutage, das aber nur während der Regenzeit genügt. Im südlichen Cota Cota (70.8/100) wird Wasser in einem Weiher gesammelt und dann in eine Ziegelei hinuntergeleitet. Quellwasser tritt in Cota Cota wenig oberhalb der 1980 trockengelegten Laguna Municipal am Straßenrand aus $(71.4 / 100.8)$ und wird dort von Leuten geholt. Hier sind überdies zahlreiche Brunnenschächte gegraben worden, die entweder als Ziehbrunnen oder mit Hand- oder Motorpumpen ihren Dienst versehen ${ }^{10}$. In Nuevo Brasil (77/85) sind zahlreiche ca. $7 \mathrm{~m}$ tiefe Brunnen, die aber in der Trockenzeit versiegen. Gegen $20 \mathrm{~m}$ tiefe Brunnenschächte gab es vor der Erschließung durch SAMAPA auch in Calacoto (71/98). Hausversorgung durch eigene Brunnen bzw. Schächte findet sich längs der Avenida Buenos Aires (75/915). Die tiefsten Brunnen sind auf dem Altiplano gelegen, wo GEOBOL für mehrere Industriebetriebe in $60-70 \mathrm{~m}$ Tiefe Wasser erbohrt hat (Abb.4). 


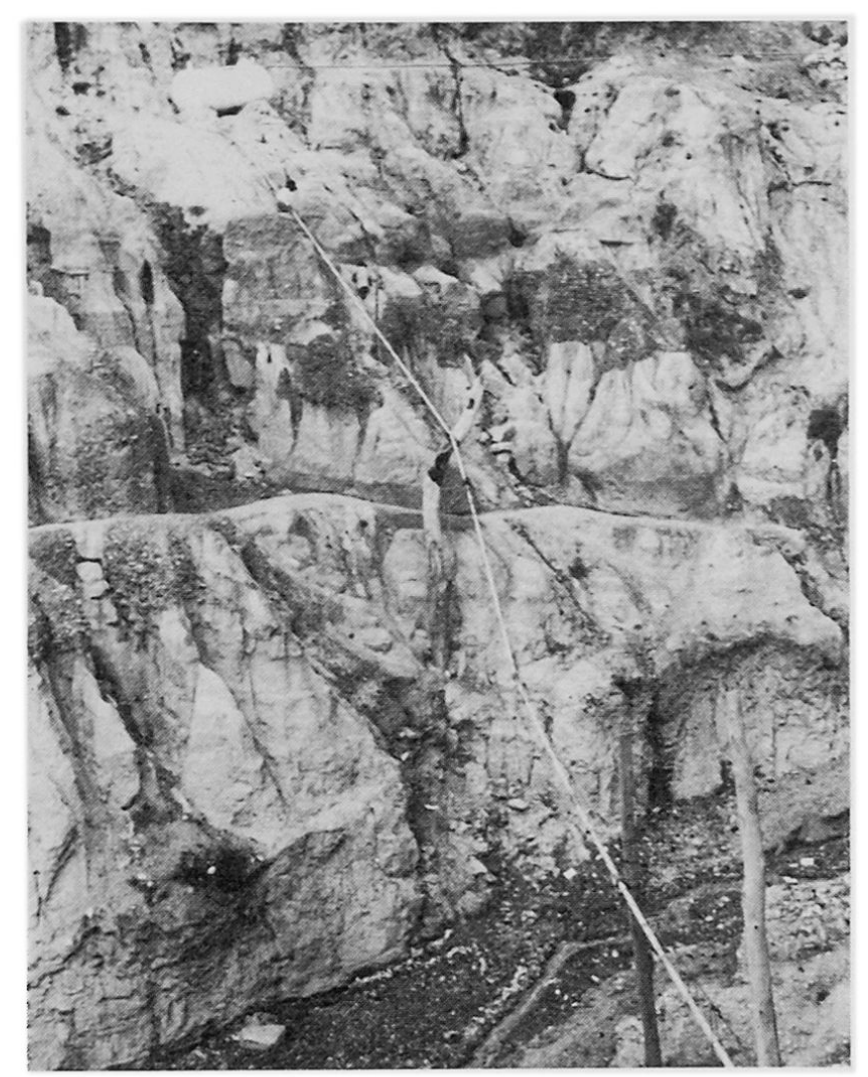

Abb. 3 Genossenschaftlich installierter Blechtank und Wasserleitung, die eine Schlucht im SE von Tacagua überspannt (73.8/92.3).

\subsection{Fehlende geregelte Wasserversorgung}

In großen Stadtteilen auf dem Altiplano ist die Wasserversorgung noch nicht sichergestellt. Diese locker überbauten Flächen sind wohl durch Straßenzüge erschlossen, haben allenfalls Kirchen, Schulen oder Buslinien, sonst aber fehlt ihnen praktisch jede Infrastruktur. In diesen Gebieten autochthoner Versorgung holen sich die Leute in Bächen Wasser oder tragen es von weit her. In einzelnen Fällen sind Blechfässer zum Sammeln von Regenwasser unter die Dachtraufe gestellt worden. Mehrere kleine Quartiere ohne Trinkwasser finden sich verstreut in der Stadt. Meist betrifft es schlechte Terrains in Bacheinschnitten, an zerfurchten Hängen oder abseits von Straßen. Damit wird angedeutet, daß sich die naturlandschaftlichen Voraussetzungen oft im sozialen Aufbau einzelner Quartiere und ihrer Wasserversorgung widerspiegeln. Mit diesen Zusammenhängen befaßt sich das folgende Kapitel.

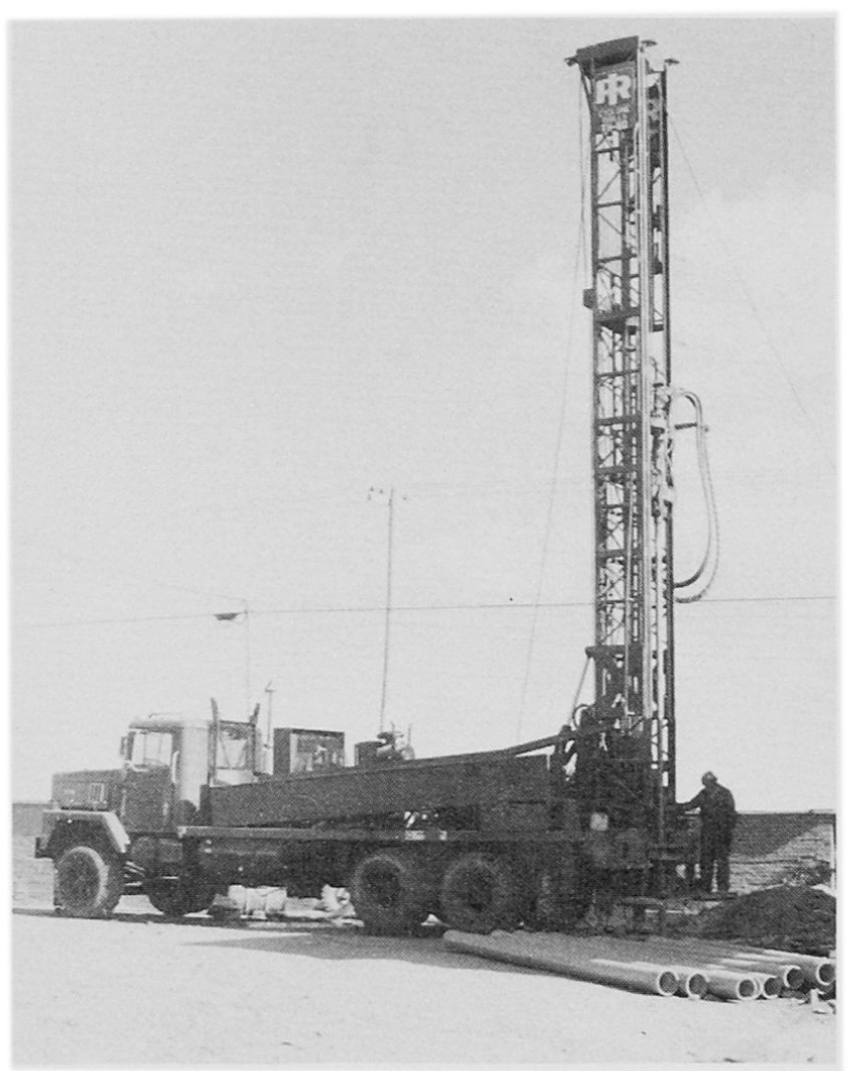

Abb. 4 Diese Bohrequipe von GEOBOL arbeitet hier auf dem Altiplano (77.5/84) und wird in ca. $70 \mathrm{~m}$ Tiefe Grundwasser erschließen.

\section{Zusammenhänge zwischen Naturlandschaft, sozialer Struktur und Wasserversorgung}

\subsection{Besonderheiten einiger Stadtteile}

Anhand der Fig. 3 versuchen wir zu analysieren, wie in $\mathrm{La} \mathrm{Paz}$ die verschiedenen Trinkwasserareale angeordnet sind. In dieser Karte wurden die Signaturen möglichst so abgestuft, daß dunkle Raster eine bessere Wasserversorgung beinhalten als helle. Als «bessere» Versorgung verstehen wir - ohne dies im speziellen Fall belegen zu können - einen Wasserbezug, der relativ höherem Wohnanspruch und Lebensstandard gerecht wird. Das enge Nebeneinander von dunkeln und hellen Flächen deutet also darauf hin, daß oft gut erschlossene Gebiete mit mangelhaft versorgten kleinräumig abwechseln. Das Gesamtbild der Stadt prägen indessen einige große, einheitlichen Flächen. Auf dem Altiplano wächst die Stadt in breiter Front gegen SW und W, aber weder Überbauung noch Wasserversorgung sind geregelt. Es sind Armenquartiere, wo ganze Sippen zusammenleben und jeder mit 
ড્

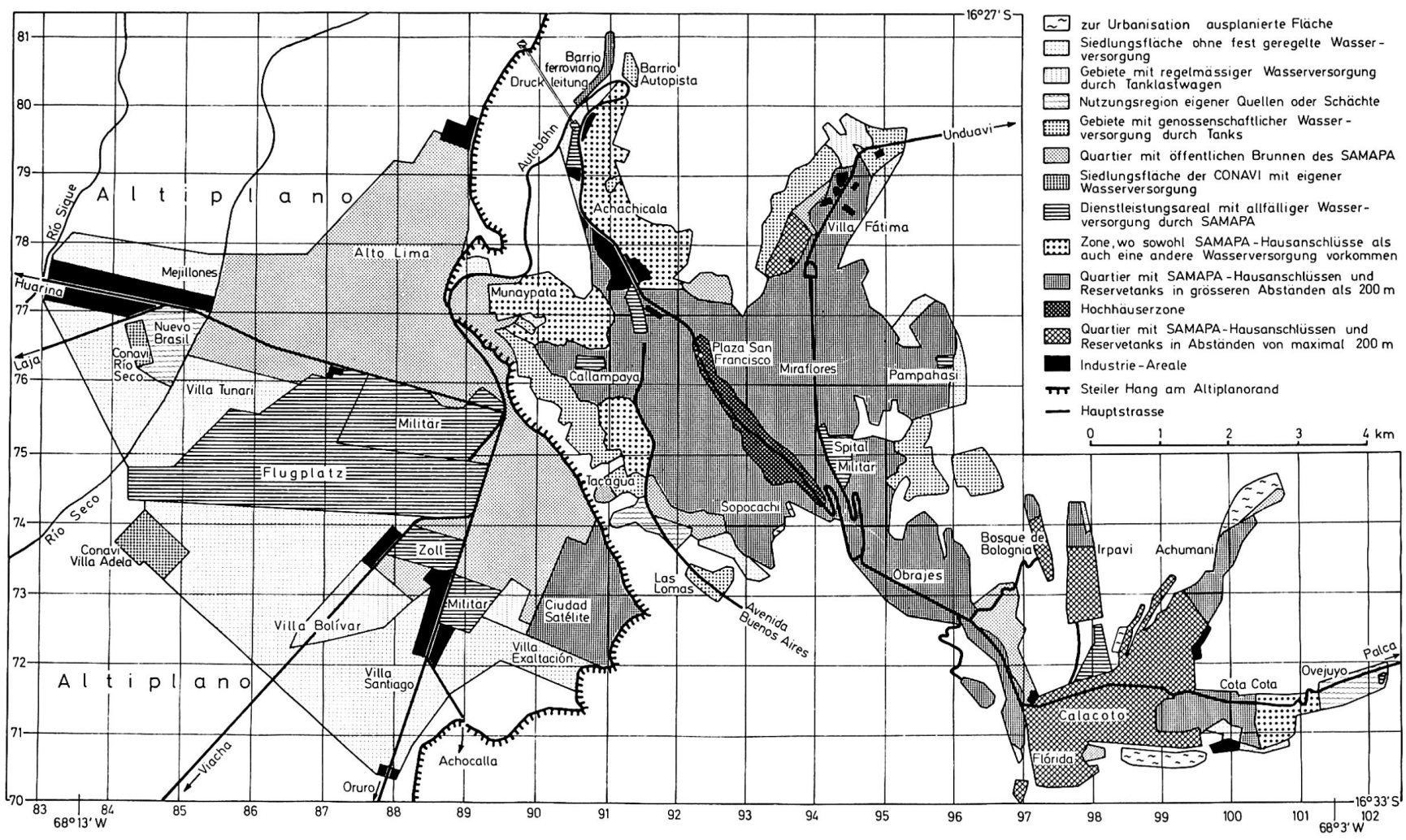

Fig. 3 Areale verschiedener Wasserversorgung in La Paz (1981) 
einigen Einkünften dem Haushalt beisteuert. Eine eigentliche Verslumung wie in lateinamerikanischen Millionenstädten findet hier aber nicht statt. Große Flächen nehmen auf dem Altiplano die Flugplatzund Militäreinrichtungen ein; ihre Wasserversorgung ist durch Reservetanks auf Gebäuden und Wassertürme gewährleistet. Nordwärts und ostwärts schließt die Zone der öffentlichen Brunnen an, ein Gebiet häufiger Wasserknappheit. Die zentralen Teile des Talkessels und die ostwärts anschließenden Quartiere hat SAMAPA weitgehend mit Hausanschlüssen versehen. Hier sind die Klimabedingungen primär besser als in den höhergelegenen Zonen, und das Angebot an Dienstleistungen ist vielseitig, was zur Selektion sozial gut gestellter Gruppen geführt hat.

In sämtlichen Quartieren kommt es mehrmals im Jahr vor, $\mathrm{da} ß$ kein Wasser fließt. Es wird bei verschiedensten Problemen abgestellt, und zwar für einige Stunden bis mehrere Tage und ohne Vorankündigung. Häufig sind Sackungen, Rutschungen oder Murgänge die Ursache von Leitungsbrüchen. Auch technische Probleme treten auf, z. B. im Zusammenhang mit der Wasseraufbereitung. Besonders in der Regenzeit stellt die Reinigung des Wassers hohe Anforderungen; eine Abfalldeponie oder ein WC im Freien können von Hochwassern durchspült werden und schlechte Wasserqualität oder Krankheiten hervorrufen. Auch zur Strafe kann SAMAPA die Wasserzufuhr unterbrechen. An der Av. Buenos Aires (74.5/91.5) haben z. B. Busfahrer an der Endstation häufig ihr Fahrzeug gewaschen; damit sie nicht mehr Wasser veschwenden können, liefert dort der öffentliche Brunnen nur noch morgens und abends während je 3 Stunden Wasser. Bei periodischer Wasserversorgung kommt es oft zu langen Warteschlangen, oder man holt sich Wasser mitten in der Nacht. Einen regelmäßigen Wassernachschub gewährleisten einzig Reservetanks auf Hausdächern, was allerdings zusätzliche Baukosten verursacht. In Fig. 3 wurde die Verbreitung dieser Reservetanks festgehalten. Ihr Inhalt schwankt zwischen ca. 1 und $4 \mathrm{~m}^{3}$. Falls sie in geringeren Distanzen als $200 \mathrm{~m}$ voneinander vorkommen, und dies in mindestens $500 \times 500 \mathrm{~m}$ großen Gebieten, so sind sie in Fig. 3 gesondert vom übrigen Gebiet mit Hausanschlüssen kartiert. Eine solche Häufung von Reservetanks existiert in den untersten Quartieren Calacoto, Achumani, Irpavi und Bosque de Bolognia (71-74/97-100), also in vornehmen Wohnvierteln (Abb.5). Hier gibt es aber auch ausgesprochen oft Wasserknappheit, da das umliegende Gelände sehr instabil ist (DOBROVOLNY 1962, Signaturen III und IV in Plates 4 und 5) und oft Sackungen die Hauptwasserleitung beschädigen. Zudem herrscht hier ein arides Klima, was während der Trockenzeit das tägliche Besprengen von Gärten bedingt. Es ist also verständlich, $\mathrm{da} ß$ sich Reservetanks in den tiefstgelegenen Quartieren häufen. Interessant ist dabei die zusätzliche Installation von Sonnenkollektoren, wie sie in jüngster Zeit auf etlichen Hausdächern in den vier genannten Quartieren vorgenommen worden ist (Abb. 5).

Zusammenfassend halten wir fest, daß im ganzen Stadtgebiet vielfältige Wasserprobleme herrschen und verschieden zu lösen versucht werden. Selbst in Hochhäusern fehlt die Kontinuität der Versorgung, weil das Wasser aus Spargründen plötzlich um $23 \mathrm{Uhr}$ abgestellt werden kann. Die Bereiche und Möglichkeiten der Wasserversorgung sind sehr vielschichtig und sollen im folgenden einer generellen Betrachtung unterzogen werden.

\subsection{Analyse einiger allgemeiner Aspekte der Wasserversorgung}

Die Agglomeration La Paz wächst und erweitert gestaffelt ihre Wasserversorgung. Die etappenweise Urbanisation geschieht v.a. an zwei Fronten: Oben bzw. im W sind es Armenviertel, und in den östlichen Quartieren unten wachsen gute Wohnquartiere in vollständig neu erschlossene Gebiete hinaus. Es stellt ein politisches Problem dar, welcher der beiden Fronten bei der Errichtung neuer Wasserleitungen erste Priorität eingeräumt wird. Dies ändert denn auch häufig bei Regierungswechseln.

In La Paz ist es nicht leicht, eine generelle Tendenz im Aufbau des Versorgungsnetzes zu erkennen. Versuchen wir dennoch, die schrittweise Erweiterung und Verbesserung mit einer ersten Hypothese zu erfassen. Denkbar ist eine Sukzession, die von einer fehlenden Wasserversorgung ausgeht und vorerst zu Tanks privater Genossenschaften führt. SAMAPA schaltet sich ein und installiert als erstes öffentliche Brunnen. In einer späteren Phase müssen Abwasserleitungen erstellt werden, und dann können Hausanschlüsse auf freien Entscheid der einzelnen Hauseigentümer eingerichtet werden. Schließlich erfaßt man damit ganze Straßenzüge und Quartiere. Eine solche Abfolge trifft durchaus für einzelne Teile von Achachicala (79/91) und Villa Fátima (78.5/94) zu. Sie vollzieht sich aber oft lückenhaft oder beschleunigt, weil besondere soziale Gegebenheiten mitspielen. So ist es zum voraus nicht in jedem Quartier gegeben, daß sich überhaupt Genossenschaften aus privater Initiative bilden. In anderen Fällen, z.B. westlich von Sopocachi (74.5/92), wehren sich die Bewohner vehement gegen die Stillegung ihrer öffentlichen Brunnen, obschon viele bereits ihre eigenen Hausanschlüsse haben. Sie gehen im Freien Wasser holen, da es ihnen billiger zu stehen kommt als zuhause. Aus diesen Erfahrungen heraus sieht SAMAPA in Munaypata (77/90) zunächst von der Zwischenlösung mit öffentlichen Brunnen ab. Sobald sie installiert sind, geben sich die Anwohner ja mit dieser Wasserversorgung zufrieden. Umgekehrt lehnen die Leute z.B. in Achumani (73.5/100) eine vorläufige Einrichtung öffentlicher Brunnen als zu primitiv ab. Bei den dortigen Urbanisationen erstellt man in jedem Neu- 


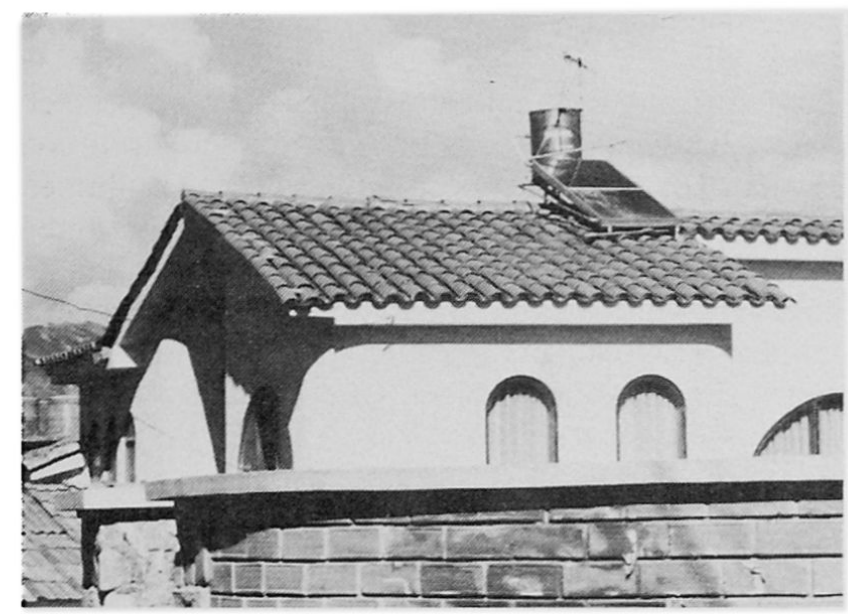

Abb. 5 Auf diesem Haus in Achumani (73.5/100) sind ein Faß als Reservetank und ein Sonnenkollektor angebracht.

bau Wasserleitungen und Kanalisation. Es zeigt sich also, daß unsere Hypothese wohl einen zunehmenden Standard in der Wasserversorgung nachzeichnet, aber daß diese in der Praxis selten mehrere Systeme schrittweise durchläuft.

Versuchen wir, mit einer zweiten Hypothese, die Unterschiede in der Wasscrversorgung zu erklären. Wir gehen von der Nachfrage aus und postulieren, $\mathrm{da} ß$ eine deutliche Steigerung des Wasserbedarfs in einem Stadtteil den Ausbau des dortigen Versorgungssystems auslöst. Im Spezialfall neuer Urbanisationen erfolgt dieser Ausbau in Quartieren der Unterschicht erst nach dem Bezug der Häuser, in guten Wohnvierteln dagegen vorher. Zur Prüfung dieser Hypothese müssen wir die Wassernachfrage abschätzen. Sie richtet sich einerseits nach der Bevölkerungsdichte (sch OоP 1980, Fig. 17a), die im Stadtzentrum am größten ist ( $>150$ Einw./ha, Versorgungszone $B)$, in den Altiplanoquartieren (Zone A) mittel und in den gehobenen Wohnquartieren am geringsten $(<50$ Einw./ha, Zone C). Anderseits ist die Wassernachfrage stark vom Lebensstandard der Leute abhängig. In Zone $A$ muß also der Wasserverbrauch einzig den Grundbedüfnisbereich abdecken, hingegen kommen extrem in Zone $\mathrm{C}$ zahlreiche weitere Verwendungen von Wasser dazu (Dusche, Schwimmbecken, Waschmaschine, Autowaschen, Gartenbesprengung). Mit diesen Ansprüchen steigt die Wassernachfrage sehr, und hierin ist sicher auch der Grund für die schnelle Integrierung ins städtische Versorgungssystem zu suchen. Die Wasserversorgung wird also in Quartieren mit geringer Bevölkerungsdichte, aber allgemein großem Wasserkonsum von SAMAPA relativ schnell eingerichtet. Es stimmt allerdings nur bedingt, da $B$ hier die Wohngebiete erst bezogen werden, nachdem

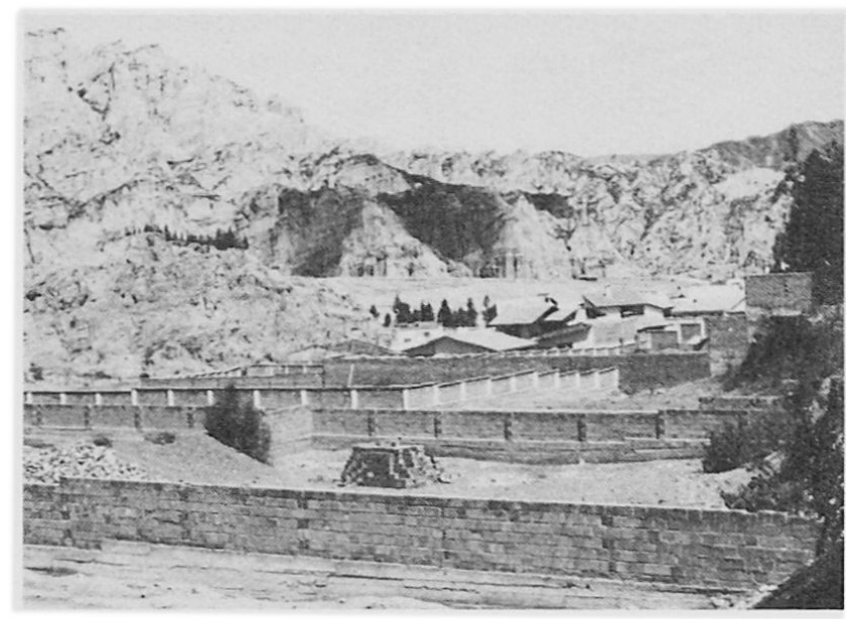

Abb. 6 Baugrundstücke am Südrand von Cota Cota (71/99.5).

die Wasserzuleitungen installiert sind. Die Baugrundstücke werden nämlich oft Jahre vor Anschluß des Trinkwassers gekauft und mit Mauern umgeben (Abb.6). Die Leute können es sich aber leisten, noch anderswo zu wohnen und die infrastrukturelle $\mathrm{Er}$ schließung abzuwarten. Im Gegensatz dazu wohnen die Leute in Armenvierteln gezwungenermaßen auf ihrem Terrain, obschon die Integrierung ins Versorgungssystem noch auf sich warten läßt.

Mit den beiden Hypothesen können einige GesetzmäBigkeiten im Wasserversorgungssystem erklärt werden, andere Fragen bleiben mangels genauer Daten offen. Immerhin erleichterten sie uns, die verschiedenen Voraussetzungen zu erkennen und diesen geographischen Faktoren Rechnung zu tragen. Aus diesen Erkenntnissen heraus können wir einige generelle Schlüsse ziehen. Die heutige Wasserversorgung von La Paz erhielt ihre Prägung durch:

- das primär von Gletschern und Niederschlägen anfallende Wasser

- die reliefbedingten Möglichkeiten

- das wechselfeuchte Klima

- die Funktion der Gebäude bzw. den Wasseranspruch

- den Zeitpunkt des Hausbaus oder der Urbanisation

- den sozialen Status der Quartierbewohner und

- im weitesten Sinn die finanziellen und technischen Möglichkeiten des Staates.

Die Wechselwirkungen dieser Faktoren sind komplex, was sich ja im uneinheitlichen Bild der Wasserversorgungskarte (Fig. 3) ausgedrückt hat. Es gilt für die Zukunft, dieses Zusammenspiel abzustimmen und es in ein klares Konzept der Wasserversorgung einzupassen. 


\section{Mögliches Konzept der zukünftigen Wasser- versorgung, Folgerungen}

Der Wasserversorgung in La Paz sind Schwierigkeiten erwachsen, die nicht kurzfristig beseitigt werden können. Man muß davon ausgehen, daß SAMAPA mit der Installation um mehrere Jahre im Rückstand liegt und die Probleme mit der Expansion der Stadt wachsen.

Ein erster Problemkreis liegt in der Finanzierung. Die hauptsächlichen Planungsstellen sind heute SAMAPA und die BID («Banco Interandino de Desarrollo» der USA), welche zur Konsultation die Firma LAMHAYER beigezogen hat. Bei der Realisierung müßte SAMAPA vermehrt die Bevölkerung beiziehen, wie dies Z. B im Barrio ferroviario (80.5/91) geschehen ist. Der Kostenanteil pro Haus betrug US\$ 230.-. Wo die Leute zu arm sind, z. B. in Tacagua (74.5/91), hat man dagegen nur einen symbolischen Beitrag von USS 0.75 pro Haushalt erhoben. Als andere Möglichkeit böten sich Fronarbeiten an, wie sie bereits bei den privaten Wassergenossenschaften geleistet werden, z. B. das Öffnen aller Gräben oder Reparaturen. In dieser Richtung wirkt auch HAM-BIRF, eine städtische Behörde zur Förderung der allgemeinen Lebensbedingungen. Sie entstand 1978 in Zusammenarbeit der «Honorable Alcaldía Municipal» von La Paz mit der "Banco Internacional de Reconstrucción y Fomento» der USA. Unter anderem fördert sie die Wasserversorgung in randstädtischen Armenvierteln. Sie gibt Kredite auf 5-15 Jahre, wobei die Nutznießer erst nach Beendigung der Bauten ratenweise zurückzahlen müssen. Es braucht oft Jahre vorsichtiger Sozialarbeit seitens HAM-BIRF, um die Leute im Gespräch zu gewinnen.

Einen zweiten Problemkreis stellt das Gesamtkonzept der Wasserversorgung dar. Das beste Verteilnetz nützt wenig, wenn es ständig durch Mängel an den Hauptleitungen außer Betrieb gesetzt wird. Ausnahmen wie die absolut gewährleistete Versorgung von Spital und Kaserne in Miraflores (75/94.5) zeigen aber, daß das Problem technisch lösbar ist. SAMAPA hat nämlich dort die beiden Versorgungszonen $\mathrm{B}$ und $\mathrm{C}$ (Fig. 2) durch eine Notfall-Leitung verbunden und kann bei Bedarf auf die Nachbarzone ausweichen. Ein ähnliches Verbundsystem müßte im ganzen Stadtgebiet angewendet werden"1. Die Hauptleitungen sind am günstigsten am Altiplanorand anzulegen. Von diesem $\mathrm{R}$ ing aus müßten radiale Verbindungsäste $\mathrm{zu}$ einer Zentrale, z. B. oberhalb Obrajes auf 3400 m (74/94.5), zusammenlaufen. Der südlichste Teil des Ringes kann allerdings nicht geschlossen werden, da die Badlands eine Verbindung über Achocalla nach Calacoto verunmöglichen. Er wäre von Villa Exaltación (72/90) direkt zur Zentrale zu schließen. Wir erachten es also als notwendig, daß in erster Priorität eine leistungsfähige Ringleitung gebaut wird von Villa Exaltación nach Tacagua (74.5/90.5), Alto Lima (78/89) ${ }^{12}$, Barrio ferroviario (81/91), Villa Fátima (79/94.5), Pampahasi
(76.3/96), Achumani (74/100) bis Ovejuyo (71.5/ 101.5). Die zweite Aufgabe des SAMAPA beschränkte sich darauf, Rohre und anderes Material bereitzustellen; die restlichen Installationen wären v. a. Sache von Zweckverbänden in den Quartieren. Dabei könnten vorderhand möglichst viele der bestehenden Formen der Wasserversorgung belassen werden.

Mit diesen Ausführungen wollten wir einige Eindrücke vermitteln, worin die Probleme beim Trinkwasser von $\mathrm{La} \mathrm{Paz}$ begründet liegen. Offenbar bringen es schon auf kleinstem Raum die sozialen Gegensätze mit sich, daß die Infrastruktur große Unterschiede aufweist. Ähnliches wie bei der Wasserversorgung beobachtet man bei anderen Infrastruktureinrichtungen, z. B. Kanalisation, Elektrizität und Gas, Kehricht, Telefonnetz, der Einrichtung von öffentlichen Märkten und der Erschließung mit Schulen, Buslinien oder asphaltierten Straßen. In diesem Bericht ging es darum, ein Beispiel dieser infrastrukturellen Ausstattung aufzugreifen und die engen Zusammenhänge zwischen dem sozialen Aufbau und dem Verteilungsmuster der Wasserversorgung zu zeigen.

\section{Resumen}

En la ciudad de La Paz/Bolivia hay diversos sistemas de abastecimiento de aqua potable. Principalmente se encuentran pilas públicas (foto 1) y carros aguateros (foto 2) en barrios de gente pobre. También se observan a veces estanques comunes de cooperativas particulares aquí (foto 3), mientras que grifos particulares normalmente están localizados en areas de la clase social media y la clase económicamente pudiente. Allí hay además tanques de reserva sobre los techos (foto 5). En la parte altiplánica de la ciudad las zonas industriales generalmente están dotadas de instalaciones propias de abastecimiento de agua porque lo han captado con perforaciones (foto 4). Mientras tanto estos contrastes no sólo resultan de diferencias sociales o económicas sino están causados por condiciones especiales del terreno con frecuencia. De esta manera varias dificultades ocurren con la reducción de la presión enorme o con derrumbes que destruyen las tuberías principales. Para mejorar el abastecimiento de agua proponemos un sistema de interconexión con tuberías principales dobles que forman un anillo cerca del borde del Altiplano. 


\section{Anmerkungen}

'Diese Grundlage erwähnt auch JORDAN (1979a, S. 36).

${ }^{2} \mathrm{Ganz}$ andere naturgeographische Voraussetzungen prägen die Stadt Mexico City, die auf Inseln im flachgründigen Lago de Texcaco gebaut worden ist (FOX 1965, S. 525-532). Seit 1900. mit der Trockenlegung des Sees und der anschließenden Übernutzung des Grundwassers, senken sich Boden und Grundwasserspiegel ständig. Daraus erwachsen enorme Probleme in der Wasserversorgung und Kanalisation.

${ }^{3}$ Herrn dipl. Natw. Christian Hanser verdanke ich die Durchsicht des Manuskripts.

${ }^{4}$ Einige Grundsätze der Kreditanstalt für Wiederaufbau schildert KARPE (1975, S.103). Zahlreiche weitere Auskünfte verdanke ich den Herren Edgar Aguilar D. und Ing. Erico Navarro A. vom SAMAPA.

${ }^{5} \mathrm{Am}$ meisten Gletscherwasser fällt zwischen Februar und August an (JORDAN 1979b, S. 306). Zwischen September und November fehlt es weitgehend, und die Regenzeit setzt erst zögernd ein, was zu einem Wassermangel in den Stauseen führt.

${ }^{6}$ Ähnliche Wasserleitungen beschreibt KINZL (1943, S. 373) in der Cordillera Blanca Perus, die hier zur Bewässerung und Trinkwasserversorgung dienen.
${ }^{7}$ Das Geld wird meistens durch Gewährsleute des Quartiers eingezogen, welche alle Brunnenbenützer kennen (z.B. eine Ladenbesitzerin). Bei einigen Brunnen gilt eine straffere Regelung: Der Wasserhahn des Brunnens ist mit einem abschließbaren Kasten versehen, und die Schlüssel gibt der Verwalter nur gegen eine Miete ab. Die monatlichen Beiträge bewegen sich zwischen USS 0.25 und 1.10 pro Person.

${ }^{8}$ Solche Angaben beziehen sich auf Fig. 3 und auf das Militärkoordinatennetz der CARTA NACIONAL BOLIVIA, 1:50000. Nach Kartenblatt 5944 müßte ausführlich ${ }^{81} 80.5 / /^{5} 91.3$ geschrieben werden.

${ }^{9}$ SCHOOP (1980, S.69) nennt sie Nachbarschaftsgruppen («juntas vecinales")

${ }^{10}$ Unbekannt ist in La Paz die ursprünglich maurische Technik der Wasserversorgung mit Qanaten. Diese unterirdischen Kanäle, die das Grundwasser anzapfen und es in schwachem Gefälle an die Oberfläche leiten, kennt man in Lateinamerika aus Mexico, Chile und Peru (sog. "puquios»; HUMLUM J., 1964/65, zit. in BRAUN 1974, S. 27).

"Eine Ringleitung gewährleistet z.B. die Wasserversorgung der Stadt Zürich und wird durch Fernsteuerung vom Wasserwerk Hardhof aus überwacht (SCHALEKAMP 1981, S. 20).

${ }^{12}$ In Alto Lima wird ab 1981 ein Werk mit 2 Becken gebaut (SAMAPA 1979, S. IV-2), das jenen von El Alto, Achachicala und Pampahasi gleichkommt.

\section{Literatur}

ANUARIOS METEOROLOGICOS (1972-1979): Klimajahrbücher des Servicio Nacional de Meteorología e Hidrología, La Paz; je ca. $100 \mathrm{~S}$.

BRAUN, C. (1974): Teheran, Marrakesch und Madrid. Ihre Wasserversorgung mit Hilfe von Qanaten. In: Bonner Geogr. Abh. 52: 1-133.

DOBROVOLNY, E. (1962): Geología del Valle de La Paz. Dep. Nacional de Geologia, Ministerio de Minas y Petroleo, Boletin No. 3 (Especial), La Paz: 1-153.

FOX, D.J. (1965): Man - water relationships in metropolitan Mexico. In: Geogr. Review, LV/4: 523-545.

GALOPPO, E. (1979): Mapa de Cobertura y Uso de la Tierra, Ciudad de La Paz (Flächennutzungskarte) 1:10 000. Programa ERTS - GEOBOL, Ministerio de Urbanismo y Vivienda, La Paz.

JORDAN, E. (1979a): Die wirtschaftliche Bedeutung der Gletscher Boliviens. In: Zeitschr. der Deutsch-Bolivianischen Industrie- und Handelskammer 1/79: 30-39.

JORDAN, E. (1979b): Grundsätzliches zum Unterschied zwischen tropischem und aussertropischem Gletscherhaushalt unter besonderer Berücksichtigung der Gletscher Boliviens. In: Erdkunde 33/4: 297-309.
KARPE, H.J. (1975): Nutzen-Kosten-Analysen in der Wasserversorgung. In: Mitt. der Versuchsanstalt für Wasserbau, Hydrologie und Glaziologie, ETH Zürich, Nr. 18: 97-114.

KINZL, H. (1943): Die anthropogeographische Bedeutung der Gletscher und die künstliche Flurbewässerung in den peruanischen Anden. Sitzungsber. europ. Geographen, Würzburg: 353-380.

KOCKS, F.H. (1967): Proyecto agua potable La Paz. Red de distribución, puntos de conexión entre las tuberias nuevas y la red existente. Bericht dieses Ingenieurbüros zuhanden SAMAPA, La Paz: 1-70, Düsseldorf.

NAVARRO, E. (1980): Abastecimiento de agua potable. Überarbeitung des Richtplans von SAMAPA (1966) 1:10 000.

SAMAPA (1979): Abastecimiento de agua potable de la ciudad de La Paz: Segunda fase, segunda etapa. In: Convocatoria No.01/81-BID, II/C: 1-200. Zu diesem mehrbändigen Werk gehören 7 Pläne 1: 5000 zum Verteilnetz von SAMAPA.

SAMAPA (1980): Informationsschrift zum XVII Congreso Interamericano de Ingenieria Sanitaria y Ambiental: $11 \mathrm{~S}$.

SCHALEKAMP, M. (1981): Die Wasserversorgung einer Großstadt am Beispiel Zürich. In: Zeitschr. "plan» 38/5: 17-22, Zürich.

SCHOOP, W. (1980): Die bolivianischen Departementszentren im Verstädterungsprozeß des Landes. In: Acta Humboldtiana, Series Geographica et Ethnographica 7: 1-319, Wiesbaden. 